\title{
Mathematical Model of the Spread of the Coronavirus Disease 2019 (COVID-19) in Burkina Faso
}

\author{
Aboudramane Guiro ${ }^{1,2}$, Blaise Koné ${ }^{2,3}$, Stanislas Ouaro ${ }^{2,3}$ \\ ${ }^{1}$ Département de Mathématiques, UFR des Sciences et Techniques Université Nazi Boni de Bobo Dioulasso, Bobo Dioulasso, \\ Burkina Faso \\ ${ }^{2}$ LAboratoire de Mathématiques et Informatique (LAMI), Ouagadougou, Burkina Faso \\ ${ }^{3}$ Département de Mathématiques, UFR des Sciences Exactes et Appliquées Université Joseph Ki Zerbo, Ouagadougou, Burkina \\ Faso \\ Email: ^abouguiro@yahoo.fr, leizon71@yahoo.fr, ouaro@yahoo.fr
}

How to cite this paper: Guiro, A., Koné, B. and Ouaro, S. (2020) Mathematical Model of the Spread of the Coronavirus Disease 2019 (COVID-19) in Burkina Faso. Applied Mathematics, 11, 1204-1218. https://doi.org/10.4236/am.2020.1111082

Received: April 5, 2020

Accepted: November 27, 2020

Published: November 30, 2020

Copyright (c) 2020 by author(s) and Scientific Research Publishing Inc. This work is licensed under the Creative Commons Attribution International License (CC BY 4.0).

http://creativecommons.org/licenses/by/4.0/

\begin{abstract}
In this paper, we develop a mathematical model of the COVID-19 pandemic in Burkina Faso. We use real data from Burkina Faso National Health Commission against COVID-19 to predict the dynamic of the disease and also the cumulative number of reported cases. We use public policies in model in order to reduce the contact rate, this allows to show how the reduction of the daily report of infectious cases goes, so we would like to draw the attention of decision makers for a rapid treatment of reported cases.
\end{abstract}

\section{Keywords}

COVID-19, Statistics, Data, Exposed Person, Reported and Unreported Cases, Mathematical Model, Public Policies, Basic Reproduction Number, Prediction

\section{Introduction}

Since December 2019, the Coronavirus disease 2019 (COVID-19) has been raving all the continents. Outbreak in Wuhan, China, the disease passed through Europe to finally reach Africa south of the Sahara. The Coronavirus disease 2019 (COVID-19) is an infectious disease that erupted in China in December 2019 and quickly spread into other countries in Europe and America. In West Africa, the first cases were reported in Senegal, Nigeria and Burkina Faso. In Burkina Faso, the first two cases were reported on March 9, 2020 ([1] [2] [3]); they were a Burkinabè couple who returned from travel from Mulhouse (France) after a few 
days stay there.

Currently, many mathematical models of the COVID-19 have been developed, mainly for the Coronavirus epidemic in China (see [1] [3]-[8]). We have developed a model describing the Coronavirus epidemic in Burkina Faso, focussing on the effects of public policies imposed by the government to contain this epidemic and the number of reported occurred cases.

\section{Mathematical Model}

Considering the known characteristics of the Coronavirus disease 2019 (COVID-19) pandemic, we assume that each person is in one of the following compartments:

- $S$ (Susceptible) means the number of persons who are not infected by the disease pathogen at time $t$, so who are susceptible to infection.

- $E$ (Exposed) represents the number of persons who are in the incubation period after being infected by the disease pathogen. These persons have no visible clinical sign of the disease. They can infect other people but with lower probability than people in the infectious comportments.

- $I$ (Infectious) means the number of persons who start developing clinical signs, these persons are asymptomatic infectious cases.

- $I_{r}$ (Reported symptomatic infectious cases) represents the number of persons who are reported and isolated at time $t$.

- $I_{u}$ (Unreported symptomatic infectious cases) stands for the number of unreported symptomatic persons at time $t$.

- $R$ (Recovered) represents a person who has survived the disease, is no longer infectious and has developed a natural immunity to the disease pathogen.

This leads to the following transfer diagram (Figure 1).

The mathematical model consists of the following system of ordinary differential equations:

$$
\left\{\begin{array}{l}
\dot{S}(t)=-\gamma(t) \frac{S(t)}{N}\left(I(t)+I_{u}(t)\right), \\
\dot{E}(t)=\gamma(t) \frac{S(t)}{N}\left(I(t)+I_{u}(t)\right)-\alpha E(t), \\
\dot{I}(t)=\alpha E(t)-\beta I(t), \\
\dot{I}_{r}(t)=\beta_{1} I-\eta I_{r}, \\
\dot{I}_{u}(t)=\beta_{2} I-\theta I_{u}, \\
\dot{R}(t)=\eta I_{r}+\theta I_{u} .
\end{array}\right.
$$

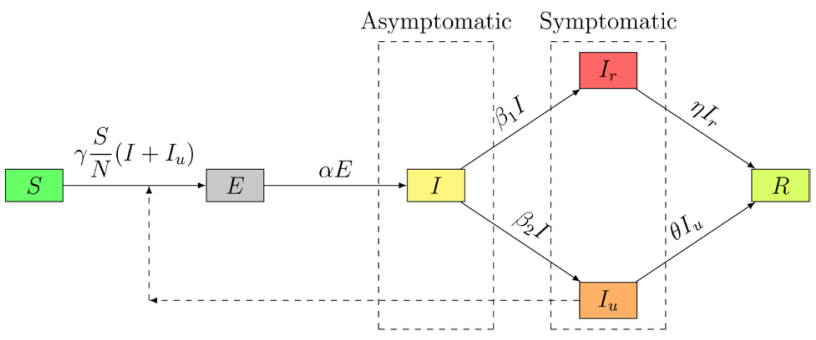

Figure 1. Transfer diagram for the mathematical model of COVID-19. 
where

- $\gamma(t)$ is the contact rate of a person in state $S\left(\mathrm{day}^{-1}\right)$ at time $t$.

- $\alpha$ is the transition rate of a person in state $E\left(\right.$ day $\left.^{-1}\right)$.

- $\beta$ is such that $1 / \beta$ is the average time during which asymptomatic infectious cases are symptomatic and $\beta_{1}+\beta_{2}=\beta$.

- $\quad \eta$ is the transition rate of a person in state $I_{r}$ to the state $R\left(\right.$ day $\left.^{-1}\right)$.

- $\theta$ is the transition rate of a person in state $I_{u}$ to the state $R\left(\right.$ day $\left.^{-1}\right)$.

- $N$ is the number of people in the territory before the start of the pandemic.

The initial data of the system is supplemented by

$$
S\left(t_{0}\right)=S_{0}>0, E\left(t_{0}\right)=E_{0}>0, I\left(t_{0}\right)=I_{0}>0, I_{r}\left(t_{0}\right)=0 \text { and } I_{u}\left(t_{0}\right) \geq 0 \text {. }
$$

The time $t$ is in day, the asymptomatic infectious individuals $I(t)$ are infectious for an average time period of $1 / \alpha$ days. We also suppose that the population is constant i.e. $N=S(t)+E(t)+I(t)+R(t)$.

Proposition 2.1. The basic reproduction number for the model system (1) is defined by

$$
R_{0}=\frac{\gamma S^{0}}{\beta N}\left(1+\frac{\beta_{2}}{\theta}\right) .
$$

Proof. We use the method of next generation matrix in [9] to compute the reproduction number $R_{0}$.

We get

$$
\mathcal{F}=\left(\begin{array}{c}
\mathcal{F}_{1} \\
\mathcal{F}_{2} \\
\mathcal{F}_{3} \\
\mathcal{F}_{4}
\end{array}\right)=\left(\begin{array}{c}
\gamma \frac{S}{N}\left(I+I_{u}\right) \\
0 \\
0 \\
0
\end{array}\right) \text { and } \mathcal{V}=\left(\begin{array}{c}
\mathcal{V}_{1} \\
\mathcal{V}_{2} \\
\mathcal{V}_{3} \\
\mathcal{V}_{4}
\end{array}\right)=\left(\begin{array}{c}
-\alpha E \\
\alpha E-\beta I \\
\beta_{1} I-\eta I_{R} \\
\beta_{2} I-\theta I_{u}
\end{array}\right) \text {, }
$$

We have

$$
\mathcal{D F}=\left(\begin{array}{llll}
\frac{\partial \mathcal{F}_{1}}{\partial E} & \frac{\partial \mathcal{F}_{1}}{\partial I} & \frac{\partial \mathcal{F}_{1}}{\partial I_{R}} & \frac{\partial \mathcal{F}_{1}}{\partial I_{u}} \\
\frac{\partial \mathcal{F}_{2}}{\partial E} & \frac{\partial \mathcal{F}_{2}}{\partial I} & \frac{\partial \mathcal{F}_{2}}{\partial I_{R}} & \frac{\partial \mathcal{F}_{2}}{\partial I_{u}} \\
\frac{\partial \mathcal{F}_{3}}{\partial E} & \frac{\partial \mathcal{F}_{3}}{\partial I} & \frac{\partial \mathcal{F}_{3}}{\partial I_{R}} & \frac{\partial \mathcal{F}_{3}}{\partial I_{u}} \\
\frac{\partial \mathcal{F}_{4}}{\partial E} & \frac{\partial \mathcal{F}_{4}}{\partial I} & \frac{\partial \mathcal{F}_{4}}{\partial I_{R}} & \frac{\partial \mathcal{F}_{4}}{\partial I_{u}}
\end{array}\right)=\left(\begin{array}{cccc}
0 & \gamma \frac{S}{N} & 0 & \gamma \frac{S}{N} \\
0 & 0 & 0 & 0 \\
0 & 0 & 0 & 0 \\
0 & 0 & 0 & 0
\end{array}\right)
$$

and

$$
\mathcal{D V}=\left(\begin{array}{llll}
\frac{\partial \mathcal{V}_{1}}{\partial E} & \frac{\partial \mathcal{V}_{1}}{\partial I} & \frac{\partial \mathcal{V}_{1}}{\partial I_{R}} & \frac{\partial \mathcal{V}_{1}}{\partial I_{u}} \\
\frac{\partial \mathcal{V}_{2}}{\partial E} & \frac{\partial \mathcal{V}_{2}}{\partial I} & \frac{\partial \mathcal{V}_{2}}{\partial I_{R}} & \frac{\partial \mathcal{V}_{2}}{\partial I_{u}} \\
\frac{\partial \mathcal{V}_{3}}{\partial E} & \frac{\partial \mathcal{V}_{3}}{\partial I} & \frac{\partial \mathcal{V}_{3}}{\partial I_{R}} & \frac{\partial \mathcal{V}_{3}}{\partial I_{u}} \\
\frac{\partial \mathcal{V}_{4}}{\partial E} & \frac{\partial \mathcal{V}_{4}}{\partial I} & \frac{\partial \mathcal{V}_{4}}{\partial I_{R}} & \frac{\partial \mathcal{V}_{4}}{\partial I_{u}}
\end{array}\right)=\left(\begin{array}{cccc}
-\alpha & 0 & 0 & 0 \\
\alpha & -\beta & 0 & 0 \\
0 & \beta_{1} & -\eta & 0 \\
0 & \beta_{2} & 0 & -\theta
\end{array}\right)
$$


On $E_{0}=\left(S^{0}, 0,0,0\right)$, we get

$$
F=\left(\begin{array}{cccc}
0 & \gamma \frac{S^{0}}{N} & 0 & \gamma \frac{S^{0}}{N} \\
0 & 0 & 0 & 0 \\
0 & 0 & 0 & 0 \\
0 & 0 & 0 & 0
\end{array}\right)
$$

and

$$
V=\left(\begin{array}{cccc}
-\alpha & 0 & 0 & 0 \\
\alpha & -\beta & 0 & 0 \\
0 & \beta_{1} & -\eta & 0 \\
0 & \beta_{2} & 0 & -\theta
\end{array}\right) .
$$

Thus, we obtain

$$
-F V^{-1}=\left(\begin{array}{cccc}
\frac{\gamma S^{0}}{\beta N}+\frac{\gamma S^{0} \beta_{2}}{\theta \beta N} & \frac{\gamma S^{0}}{\beta N}-\frac{\gamma S^{0} \beta_{2}}{\theta \beta N} & 0 & \frac{\gamma S^{0}}{\theta N} \\
0 & 0 & 0 & 0 \\
0 & 0 & 0 & 0 \\
0 & 0 & 0 & 0
\end{array}\right) .
$$

The basic reproduction number is defined as the dominant eigeinvalue of the matrix $-F V^{-1}$.

Therefore,

$$
R_{0}=\frac{\gamma S^{0}}{\beta N}\left(1+\frac{\beta_{2}}{\theta}\right)
$$

The basic reproduction number $R_{0}$ is defined as the number of cases that one infected person generates on average during his infectious period, in an uninfected population and without any special control measures. This number does not change during the spread of the disease.

The effective reproduction number $R_{e}(t)$ is defined as the number of cases that one infected person generates during his infectious period. This effective reproduction number depends on time, so, on public policies (change during the spread of the disease). Furthermore, $R_{e}(0)=R_{0}$ and the spread of the disease slows when $R_{e}(t)<1$.

Since

$$
R_{0}=\frac{\gamma S^{0}}{\beta N}\left(1+\frac{\beta_{2}}{\theta}\right)
$$

therefore

$$
R_{e}(t)=\frac{\gamma}{\beta}\left(1+\frac{\beta_{2}}{\theta}\right) \frac{S(t)}{N} .
$$

\section{Data}

In this part, we use real data generated by the Burkina Faso National Health 
Commission against Coronavirus Disease 2019 (COVID-19) called CORUS. Recall that the first cases were reported in Burkina Faso on March 9, 2020, From that date, we witnessed an exponential growth in the number of cumulated cases. Table 1 represents the daily cumulative number of reported infected cases and Table 2 the daily reported infected cases.

With these data, we can see the daily dispersion of the infected case (Figure 2 ), and the cumulative reported infected cases (Figure 3 ).

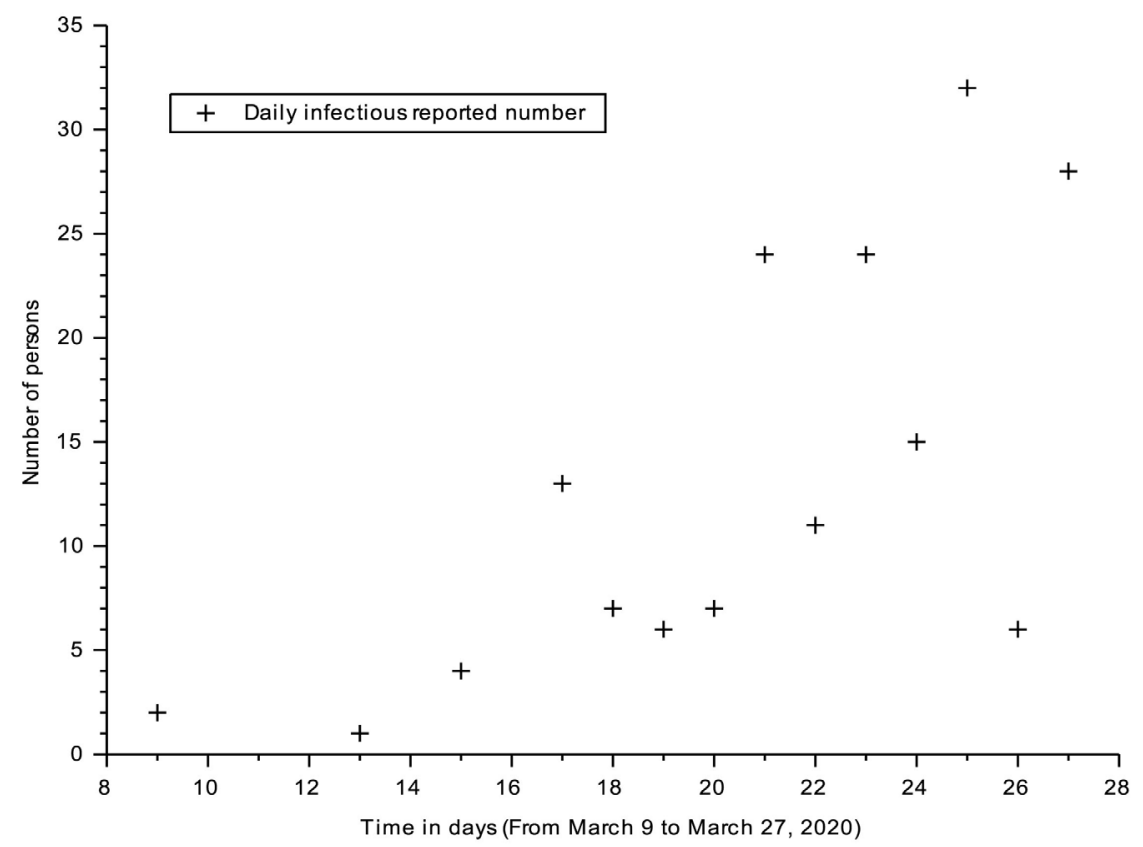

Figure 2. Daily infected reported number.

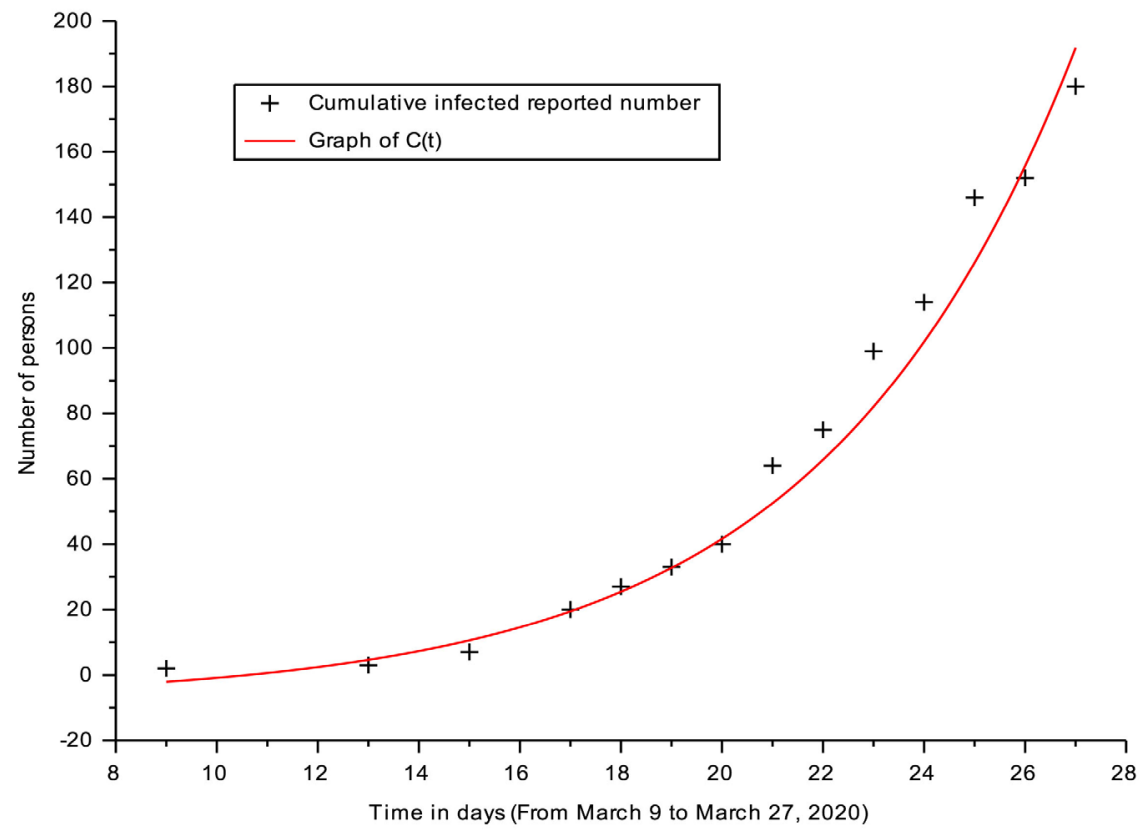

Figure 3. Cumulative infected reported number. 
Table 1. Cumulative daily reported cases data from March 9, 2020 to March 27, 2020 for Burkina Faso by Burkina Faso National Health Commission against Coronavirus Disease 2019 (COVID-19).

\begin{tabular}{ccccccccccc}
\hline March & 9 & 13 & 15 & 17 & 18 & 19 & 20 & 21 & 22 & 23 \\
\hline \multirow{2}{*}{ March } & 24 & 3 & 7 & 20 & 27 & 33 & 40 & 64 & 75 & 99 \\
& 25 & 26 & 27 & & & & & & \\
& 114 & 146 & 152 & 180 & & & & & \\
\hline
\end{tabular}

Table 2. Daily reported cases data from March 9, 2020 to March 27, 2020 for Burkina Faso by Burkina Faso National Health Commission against Coronavirus Disease 2019 (COVID-19).

\begin{tabular}{lcccccccccc}
\hline March & 9 & 13 & 15 & 17 & 18 & 19 & 20 & 21 & 22 & 23 \\
\hline \multirow{2}{*}{ March } & 24 & 1 & 4 & 13 & 7 & 6 & 7 & 24 & 11 & 24 \\
& 25 & 26 & 27 & & & & & & \\
& 15 & 32 & 6 & 28 & & & & & \\
\hline
\end{tabular}

\section{Model Parameters for COVID-19 in Burkina Faso}

Some of the parameters used in the simulations presented in Section 5 are from the literature. However, due to specificity of our real data, we fit some other parameters mainly to adjust the cumulative curve $C(t)$. This curve represents the cumulative number of reported symptomatic infectious cases at time $t$. So using the fact that $\beta=f \beta+(1-f) \beta=\beta_{1}+\beta_{2}$, we have:

$$
C(t)=\beta_{1} \int_{t_{0}}^{t} I(s) \mathrm{d} s
$$

For the beginning of the epidemic, we assume that the growth of the cumulative curve is exponential as in [10], i.e.

$$
C(t)=a \mathrm{e}^{b t}-c, \forall t \geq t_{0} .
$$

We use the cumulative number of reported symptomatic infectious cases from Table 1 to evaluate $a, b, c$ and $t_{0}$.

So from [4] and [10], we have

$$
I_{0}=\frac{b c}{f \beta}, \quad \gamma\left(t_{0}\right)=\gamma_{0}=\frac{b+\beta}{S_{0}} \frac{\theta+b}{\beta_{2}+\theta+b} \text { and } I_{r_{0}}=\frac{\beta_{2}}{\theta+b} I_{0} .
$$

With the data in Section 3, we fit the cumulative infectious cases reported number by :

$$
C(t)=0.9 \mathrm{e}^{0.2 t}-7.5,
$$

so we have $a=0.9, b=0.2$ and $c=7.5$.

\section{Simulation and Comments}

The data we use here in Table 3 were calibrated for the situation of China, the first big source of COVID-19 [1] [3] [4] [7]. Due to the spread of the disease, 
Table 3. Summary of some parameters calibrated for COVID-19 from the literature and data fit in Burkina Faso.

\begin{tabular}{|c|c|c|c|}
\hline Notation & Value & Description & Reference \\
\hline$\alpha$ & 0.1818 & Trans. rate of persons in state $E\left(\right.$ day $\left.^{-1}\right)$ & [1] \\
\hline$\eta=\theta$ & $\frac{1}{14}$ & $\begin{array}{l}\text { Trans. rate of persons in state } I_{u}, I_{r} \text { to state } \\
R\left(\text { day }^{-1}\right)\end{array}$ & [1] \\
\hline$f$ & 0.7 & $\begin{array}{l}\text { Fraction of asymptomatic infectious that become } \\
\text { reported symptomatic }\end{array}$ & fixed \\
\hline$\beta$ & $\frac{1}{7}$ & Trans. rate of a persons in state $I\left(\right.$ day $\left.^{-1}\right)$ & [11] \\
\hline$\beta_{1}=f \beta$ & 0.1 & $\begin{array}{l}\text { Rate at which asymptomatic infectious become } \\
\text { reported symptomatic }\end{array}$ & \\
\hline$\beta_{2}=(1-f) \beta$ & 0.0428 & $\begin{array}{l}\text { Rate at which asymptomatic infectious become } \\
\text { unreported symptomatic }\end{array}$ & \\
\hline$t_{0}$ & & Time at which the epidemic started & fitted \\
\hline$S_{0}$ & & Number of susceptible at time $t_{0}$ & fitted \\
\hline$I_{0}$ & & Number of asymptomatic infectious at time $t_{0}$ & fitted \\
\hline$I_{r_{0}}$ & & $\begin{array}{l}\text { Number of unreported symptomatic infectious } \\
\text { at time } t_{0}\end{array}$ & fitted \\
\hline$\gamma$ & & Transmission rate & fitted \\
\hline
\end{tabular}

new studies should be performed to analyze its behavior in other sanitary population and climatic conditions. Only a limited number of studies accepted by the scientific community are available and these are what we use in Table 3.

For the parameters used in this model, we present some important parameters and threshold values related to the Coronavirus Epidemic in Burkina Faso. In particular, we observe that the basic reproduction number $R_{0}=4.9$ is bigger than other reproduction number values reported in the literature [3] [10] [12]. This could be explained by the fact that we have taken into account unreported infected persons.

We assume that the exponential increase phase of the epidemic is intrinsic to the population of each region. Also, the Susceptible population $S(t)$ is not significantly reduced over the time. We suppose that the entire population of Burkina Faso at the date $t_{0}$ are susceptible so, $S\left(t_{0}\right)=20000000$, the exposed population at the same date $E\left(t_{0}\right)=E_{0}=200$. Applying the model to Ouagadougou, the capital city, we set $S\left(t_{0}\right)=2000000$ and $E\left(t_{0}\right)=E_{0}=200$.

\subsection{Without Any Public Policies until March 26, 2020}

We use the cumulative curve to fit the parameter $\gamma$. From the beginning of the epidemic until March 9, 2020, we assume that there was no public policy so we fit $\gamma=0.7$. This could be corroborated by the fact that the cumulative infected data curve fits well with the component of the reported infectious. Figure 4 shows the evolution of the cumulative infected reported cases and the forecast 


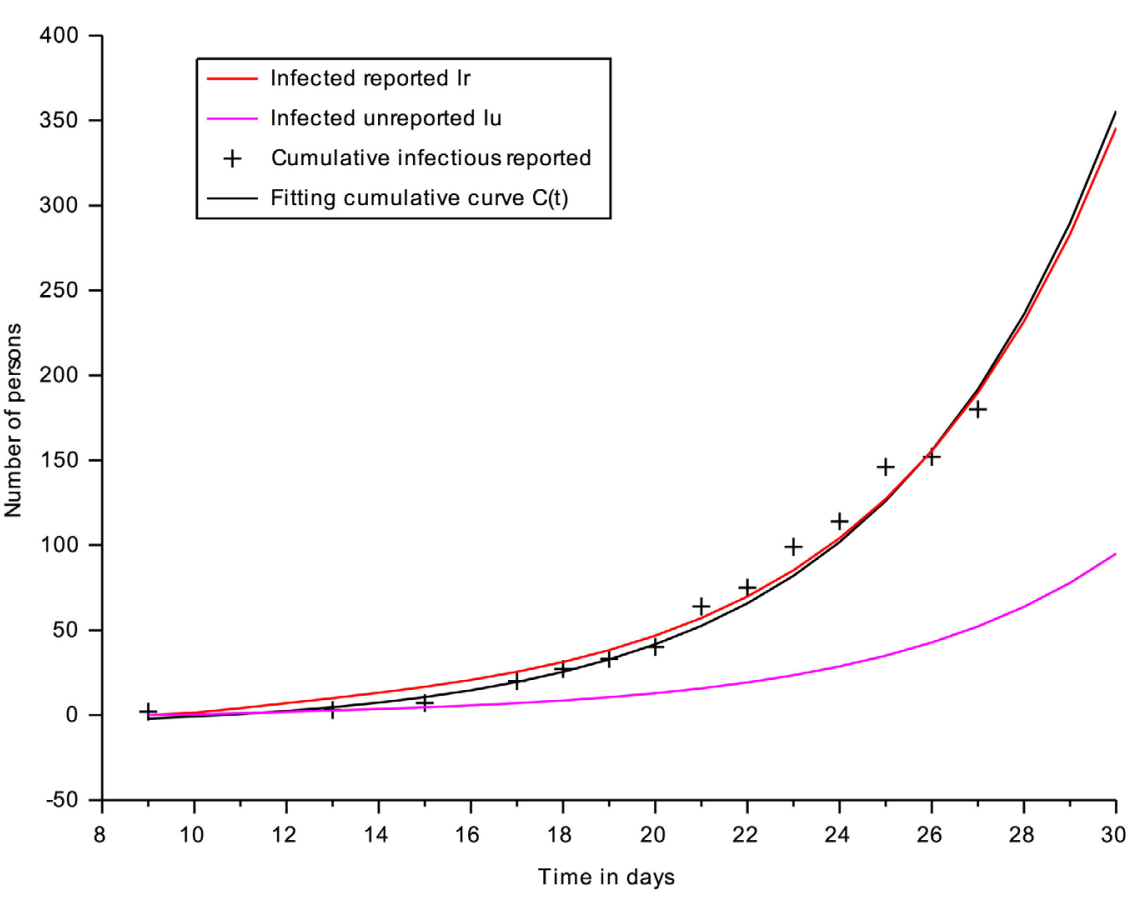

Figure 4. Fitting data with the model (1).

relating to the model. Figure 5 shows the evolutions of different types of infected people without any public policy in the country. For the entire Burkina Faso, we can reach the bar of six million infected people in the long term with five hundred thousand people in Ouagadougou as is shown in Figure 6.

\subsection{With Pubic Policies Started on March 27, 2020}

From this date, we decrease the rate of contact $\gamma(t)$ due to different types of measures taken by the public authorities. We can see the effect of these measures on the spikes of different types of infectious cases which decrease according to the degrees of the measures taken. We can see the curve of the contact rate in Figure 7.

$$
\gamma(t)=\left\{\begin{array}{l}
\gamma_{0}, \quad 0 \leq t \leq 27 \\
\gamma_{0} \exp (-\mu(t-27)), t \geq 27
\end{array}\right.
$$

Depending on the public measures taken, $\mu$ increases, so the contact rate $\gamma(t)$ decreases and it is possible to limit the number of infected persons. $\mu$ is chosen in such a way that the simulation for the time interval aligns with the cumulative reported case data. So we are able to predict the future values of the epidemic from the early cumulative reported data. The earlier the decisions public policies are made, the better the management of the epidemic. So for instance, Figures 8-13 show the evolution of different types of infectious cases in Burkina for $\mu=0.1, \mu=0.13, \mu=0.16, \mu=0.2, \mu=0.25, \mu=0.3$. Figure 14 and Figure 15 represent the evolution of the cumulative reported case when $\mu=0.05$ and $\mu=0.1$. 


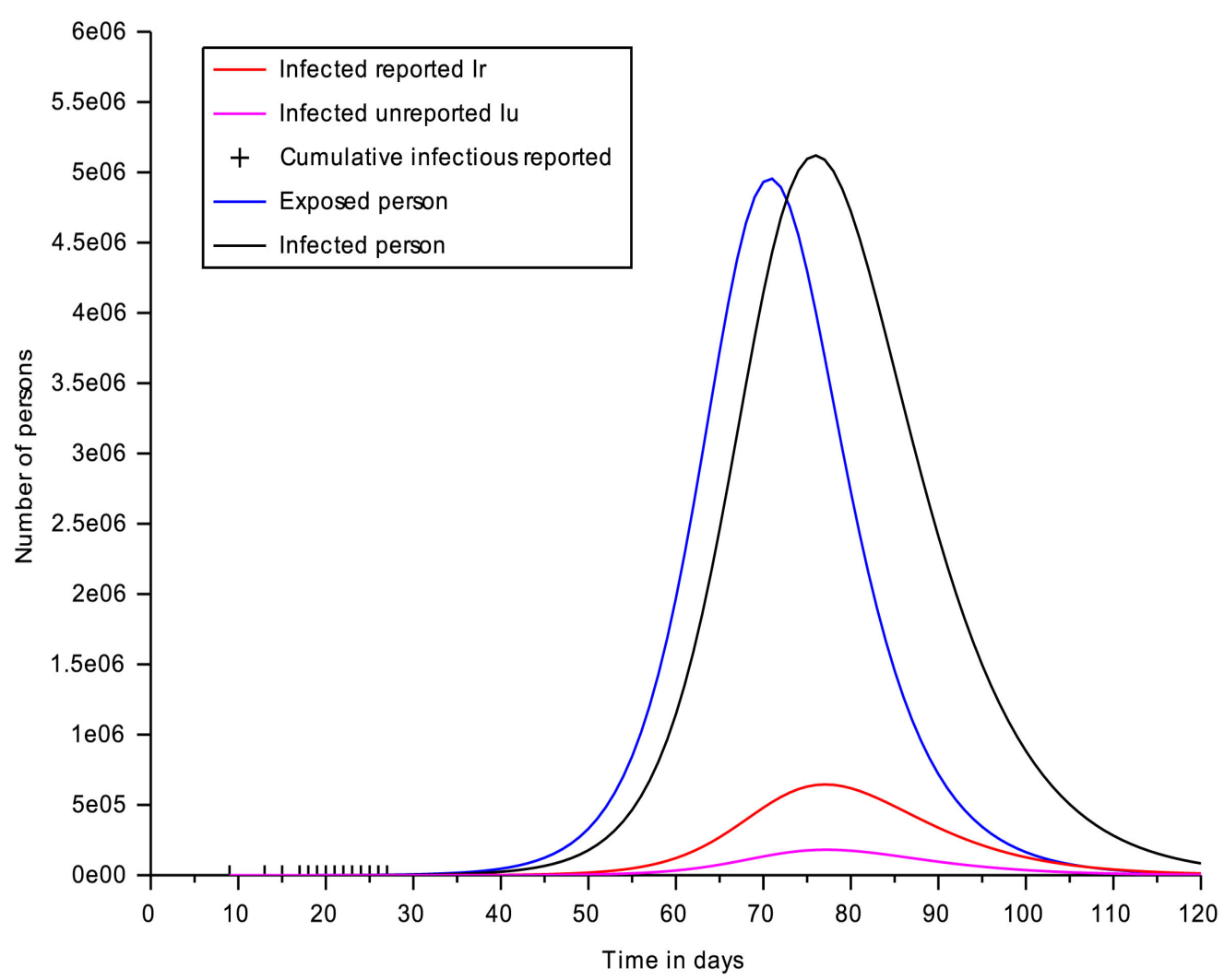

Figure 5. Forcast of the evolution of different types of infectious in Burkina.

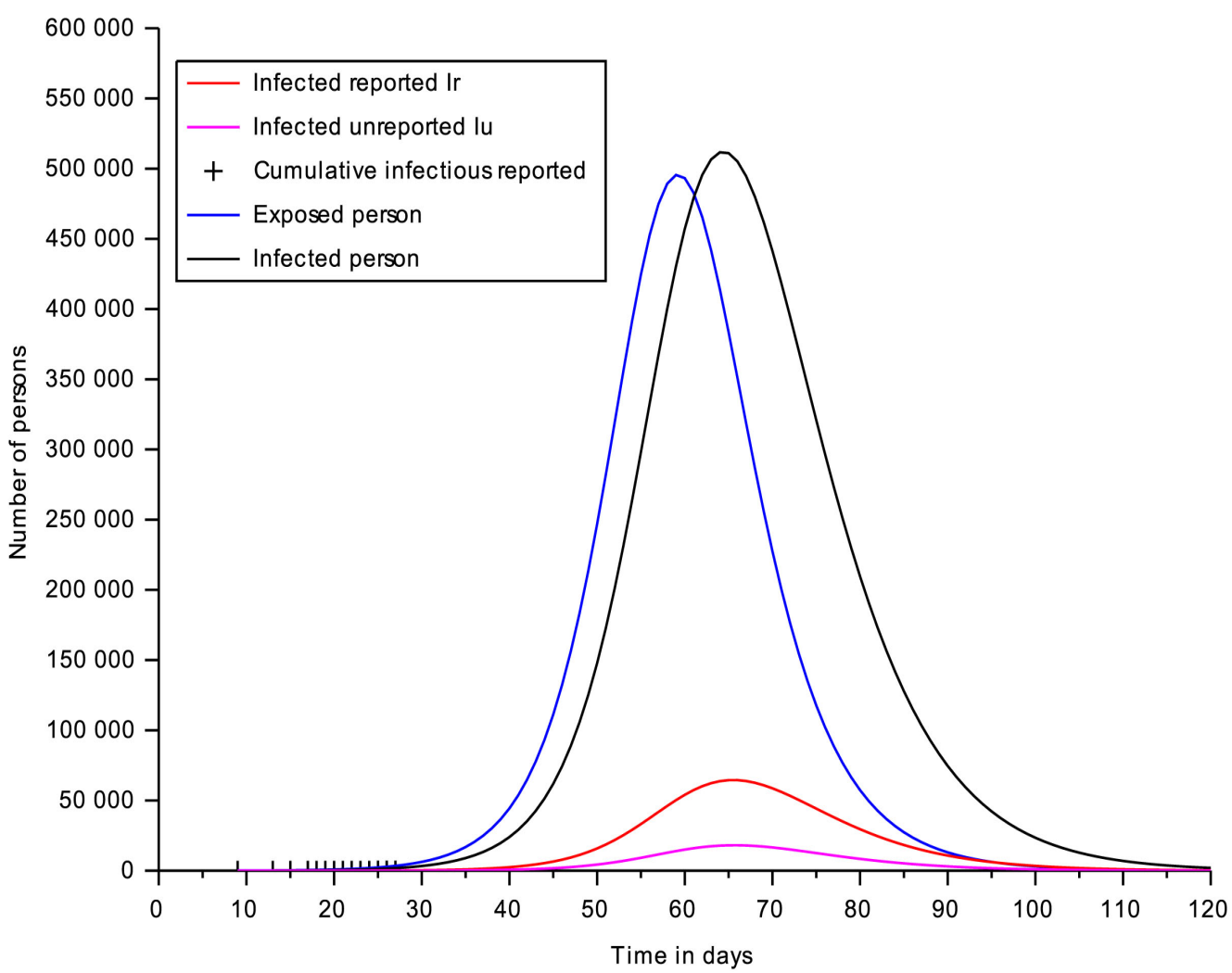

Figure 6. Forcast of the evolution of different types of infectious in Ouagadougou. 


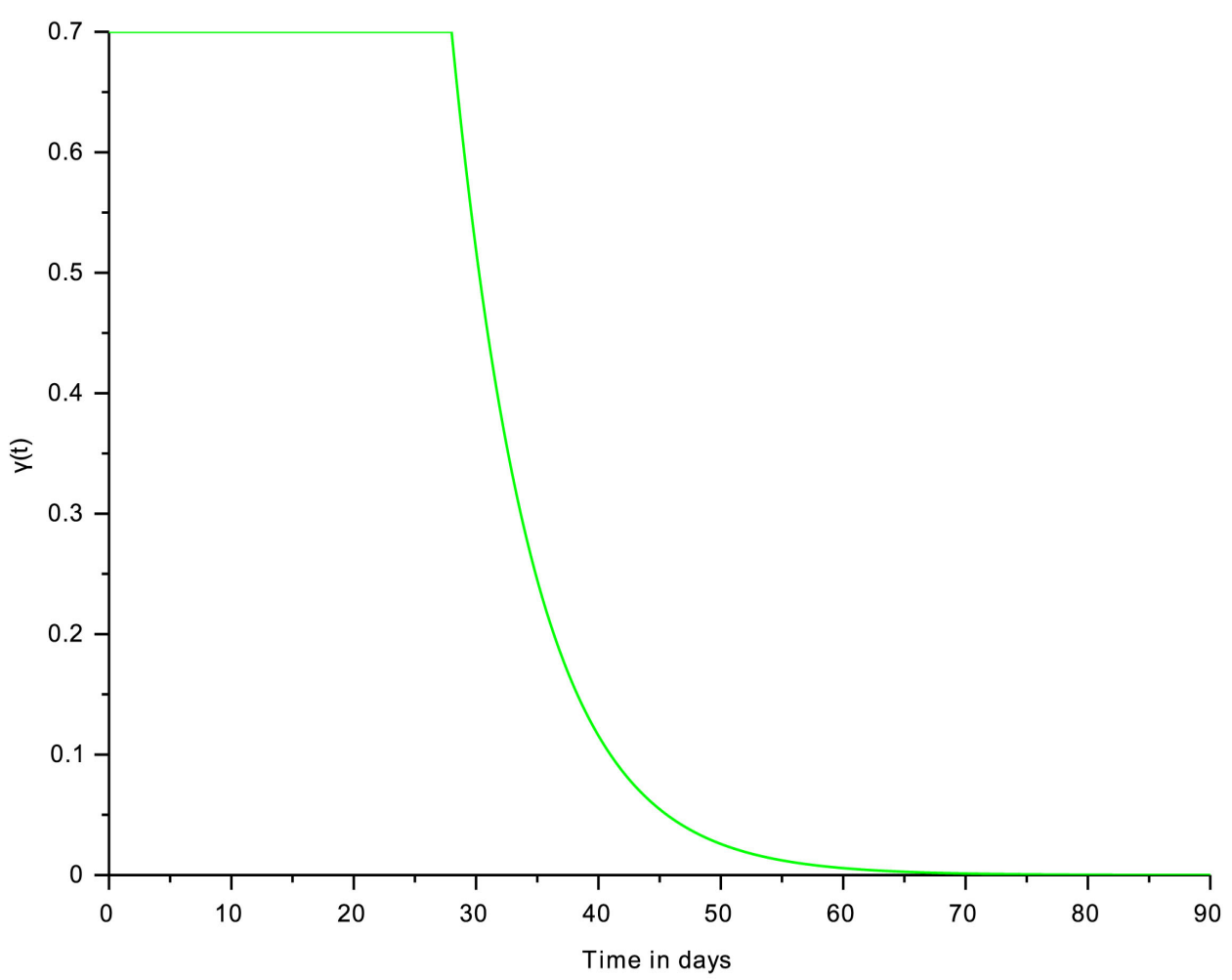

Figure 7. Contact rate $\gamma(t)$ for $\mu=0.15$.

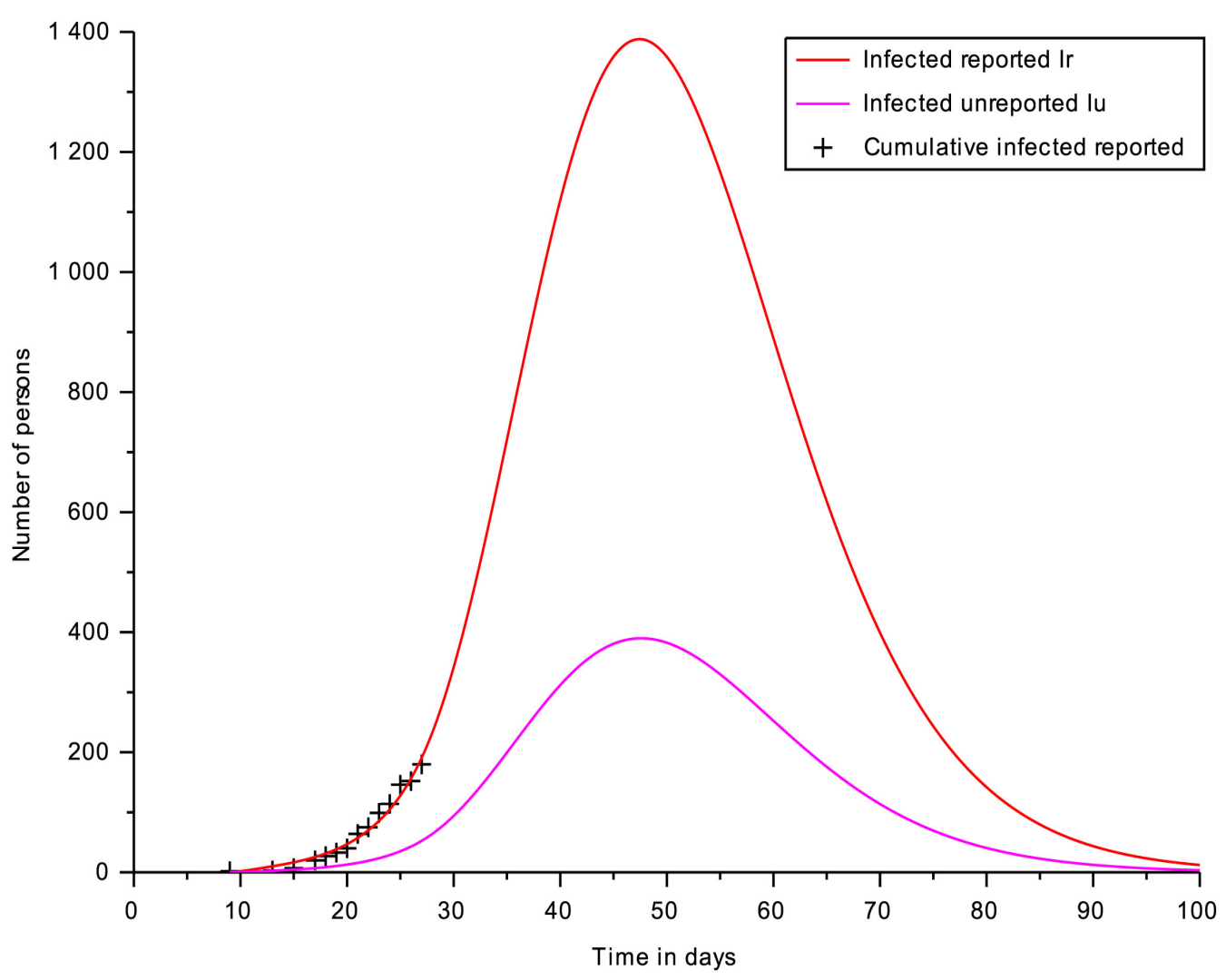

Figure 8. Forcast of the evolution of different types of infectious cases in Burkina for $\mu=0.1$. 


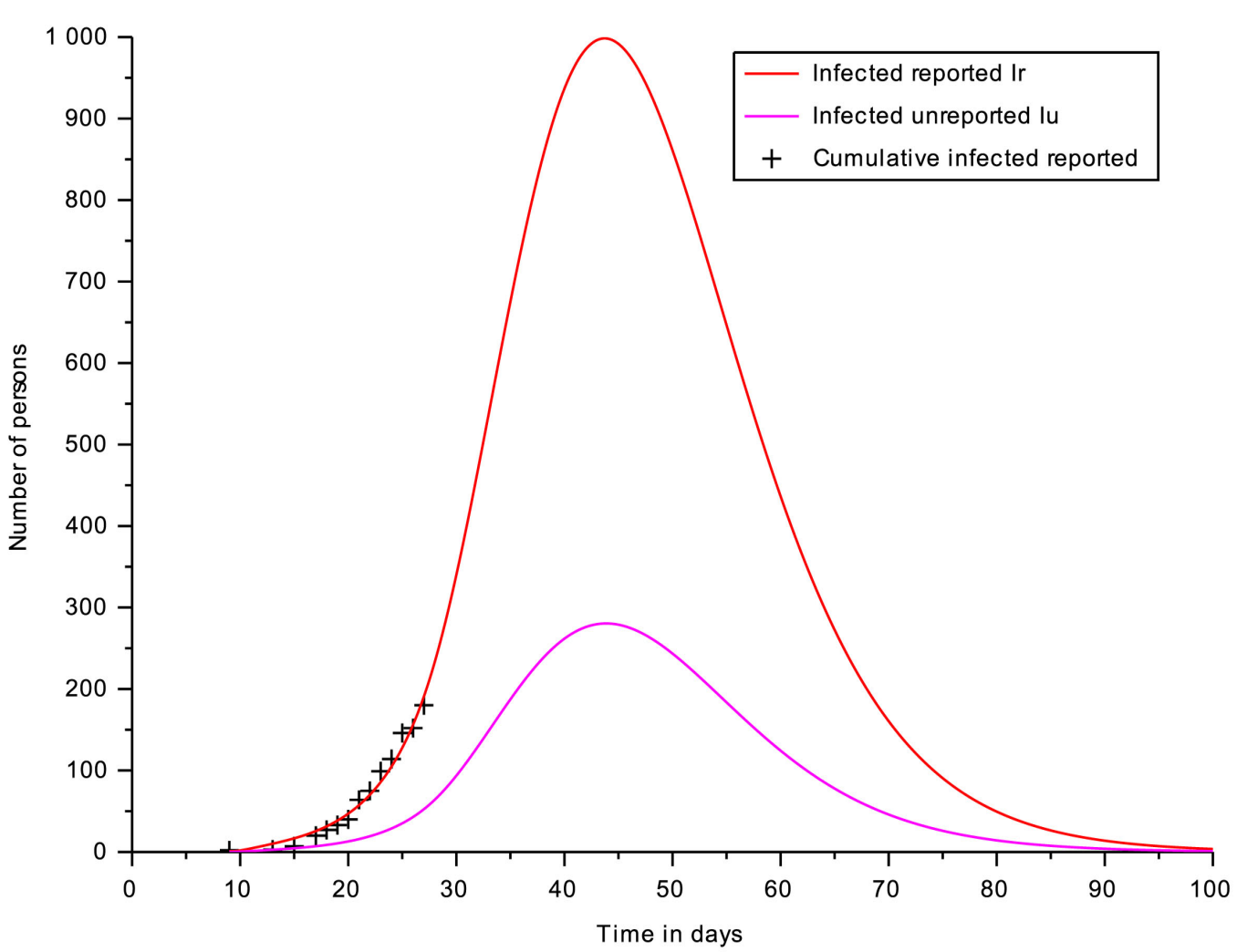

Figure 9. Forcast of the evolution of different types of infectious cases in Burkina for $\mu=0.13$.

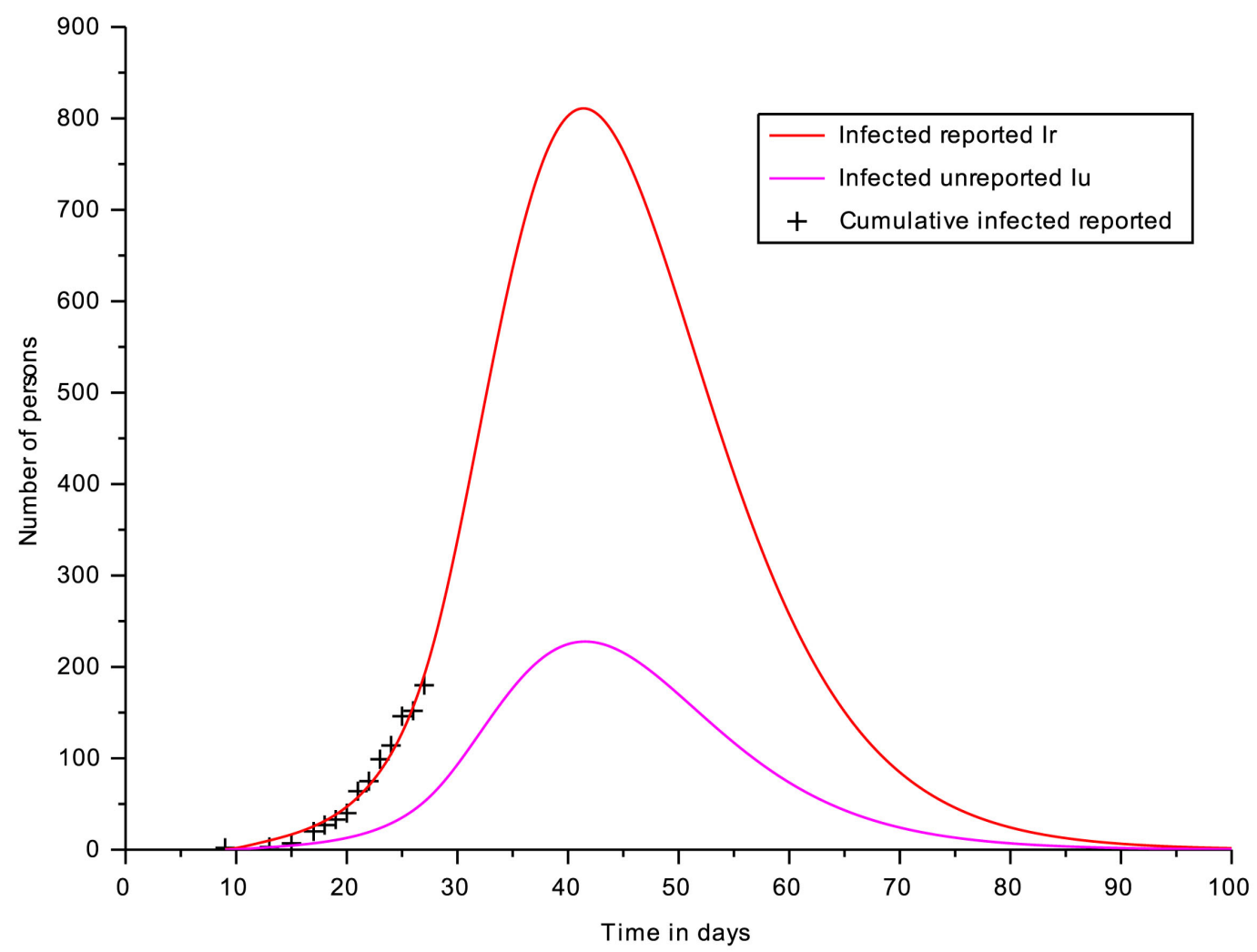

Figure 10. Forcast of the evolution of different types of infectious cases in Burkina for $\mu=0.16$. 


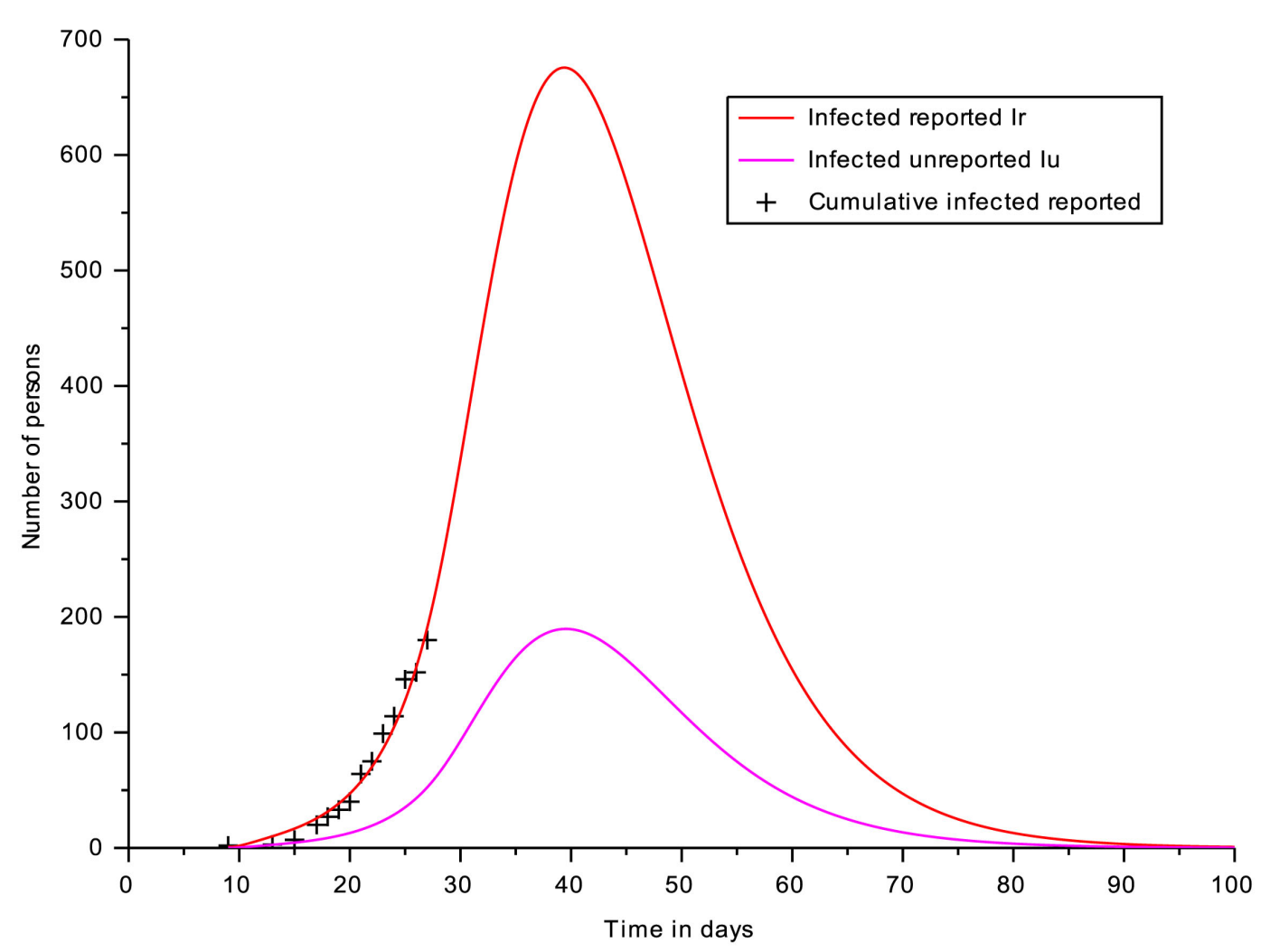

Figure 11. Forcast of the evolution of different types of infectious cases in Burkina for $\mu=0.2$.

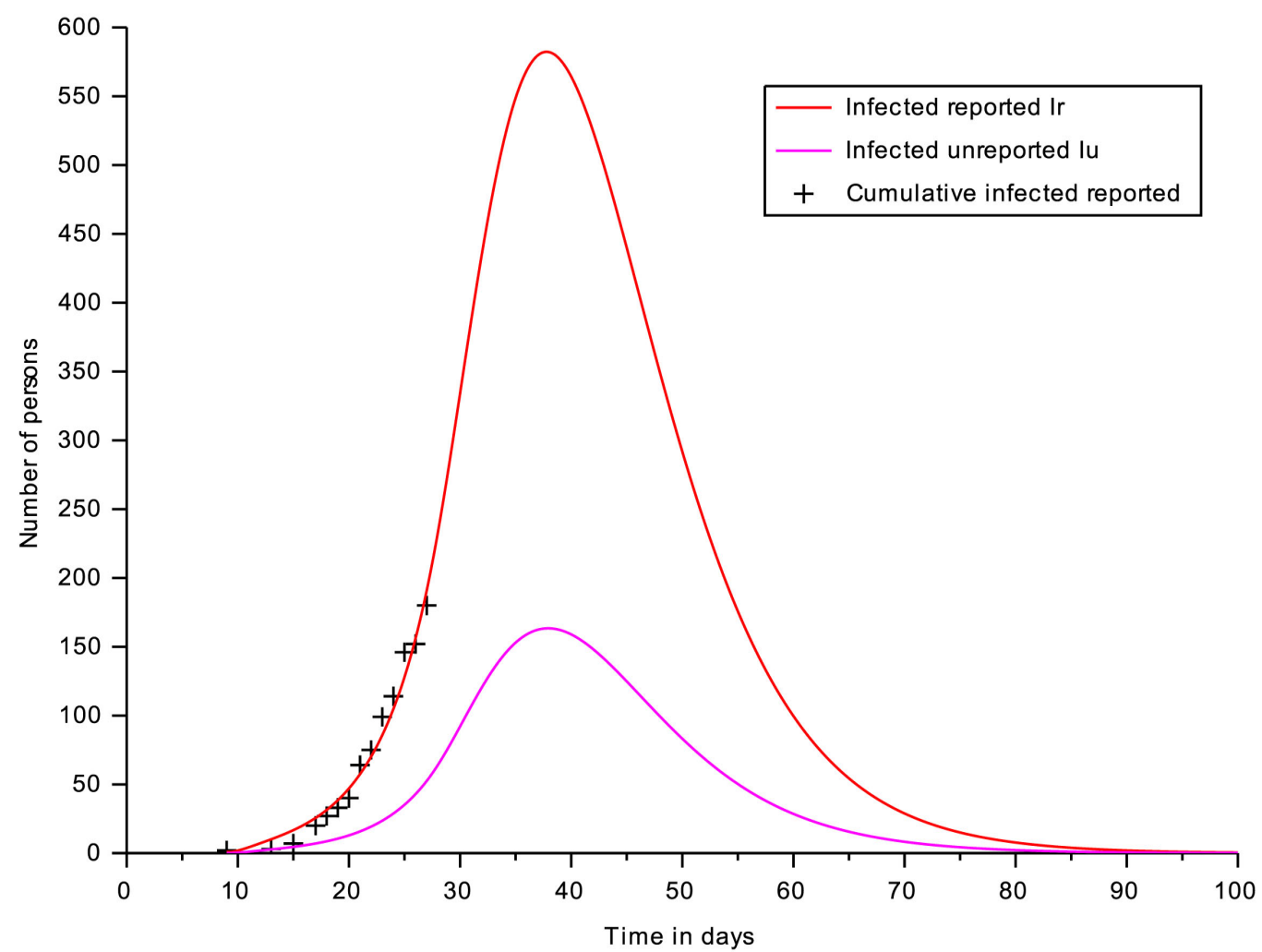

Figure 12. Forcast of the evolution of different types of infectious cases in Burkina for $\mu=0.25$. 


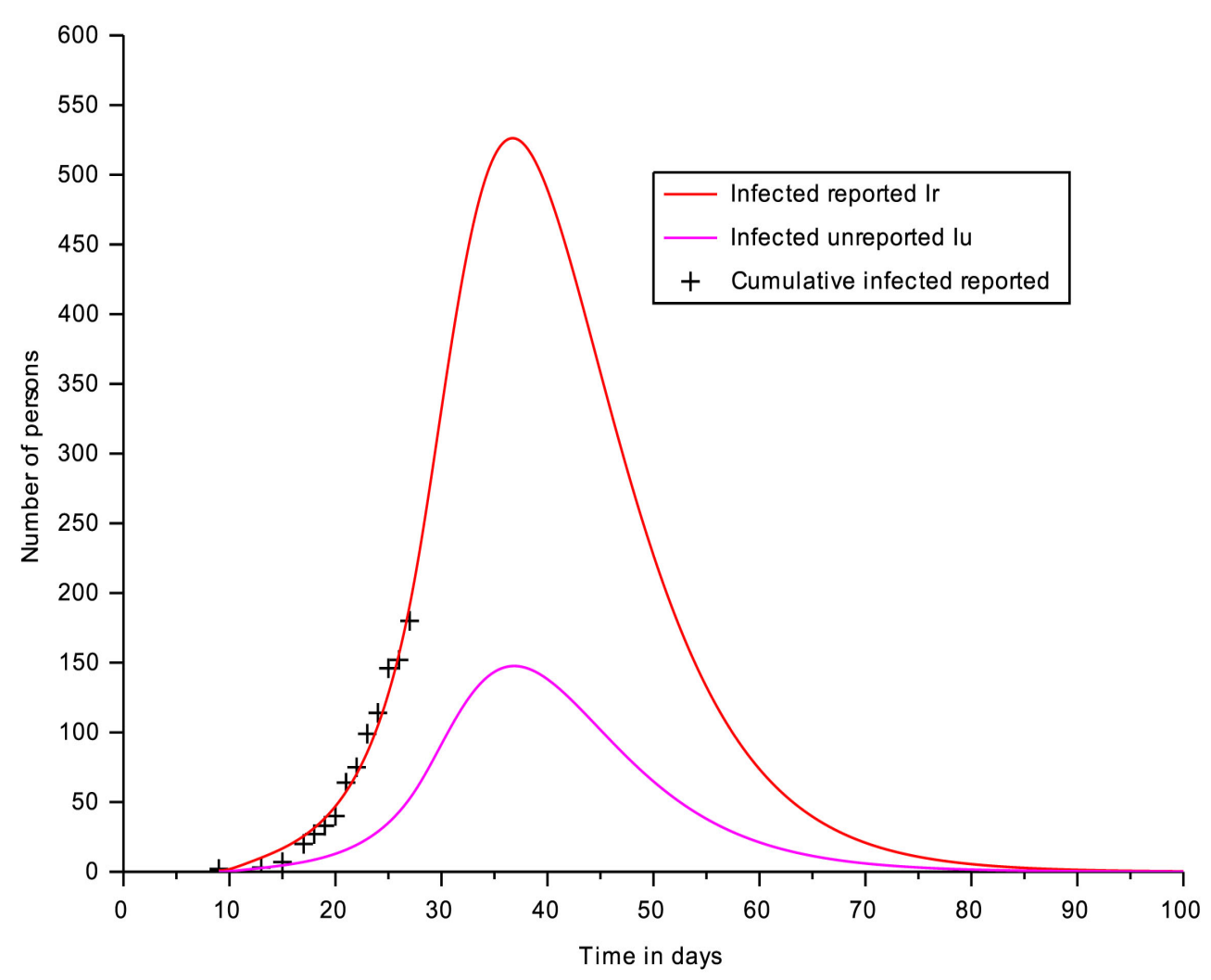

Figure 13. Forcast of the evolution of different types of infectious cases in Burkina for $\mu=0.3$.

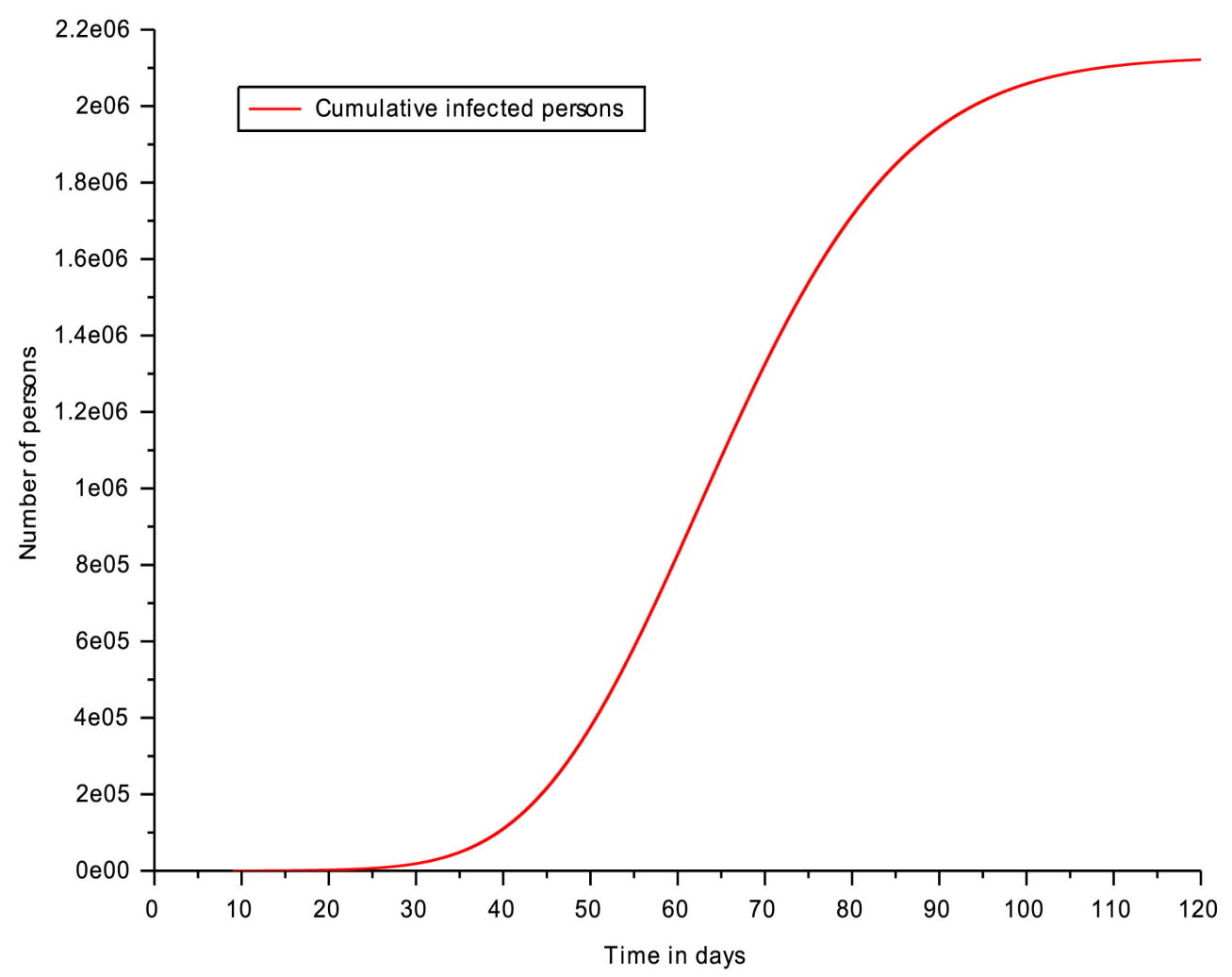

Figure 14. Evolution of the cumulative infectious cases in Burkina for $\mu=0.05$. 


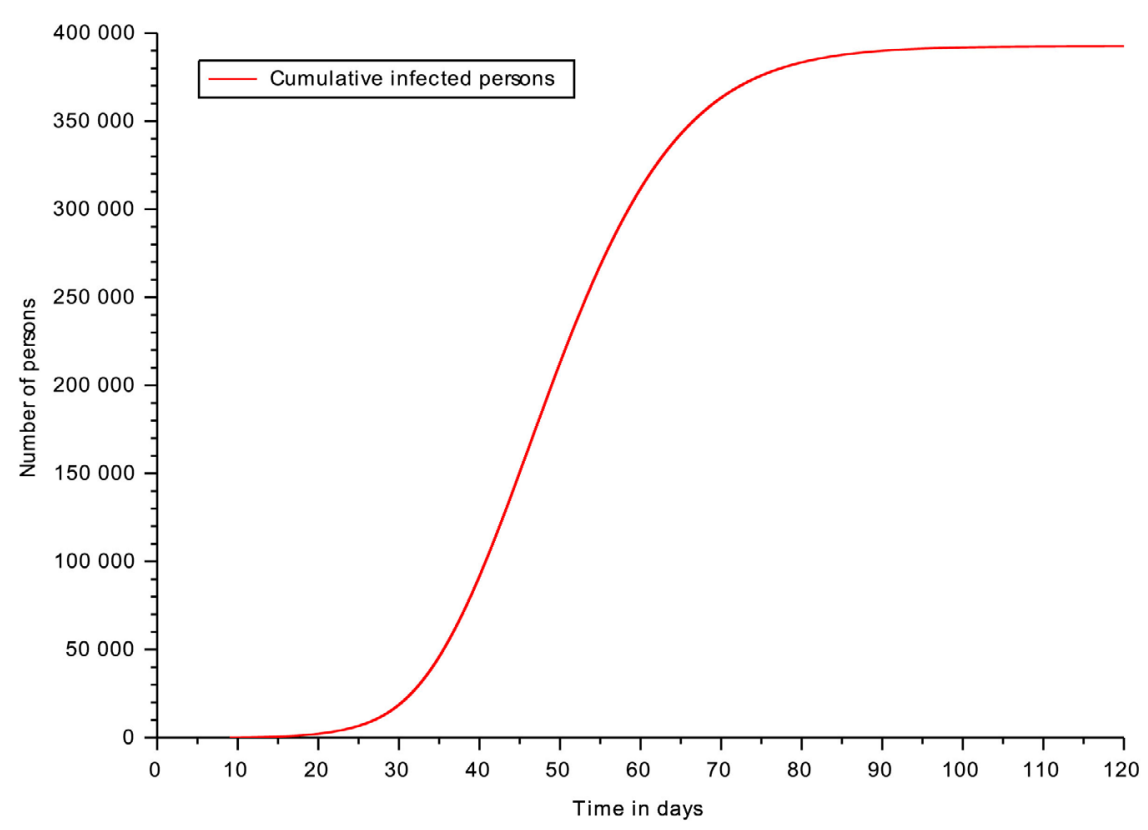

Figure 15. Evolution of the cumulative infectious cases in Burkina for $\mu=0.1$.

\section{Conclusion}

In this paper, we have developed a mathematical model of COVID-19 for Burkina Faso, inspired by models in [3] and [10]. We have been able to estimate some parameters which have made it possible to fit the model to real data from the start of the Epidemic up to March 27, 2020 (when public policies were introduced). It emerges from this model that the most important parameter here is the contact rate which is a time dependent function (with respect to the public policies taken). A drastic reduction of the contact rate can lead to a considerable reduction in the number of infectious and of the duration of the epidemic.

\section{Conflicts of Interest}

The authors declare no conflicts of interest regarding the publication of this paper.

\section{References}

[1] World Health Organization (2020) Report of the WHO-China Joint Mission on Coronavirus Disease 2019.

https://www.who.int/docs/default-source/coronaviruse/who-china-joint-mission-o n-covid-19-final-report.pdf

[2] Ivorra, B., Ramos, A.M. and Ngom, D. (2015) Be-CoDiS: A Mathematical Model to Predict the Risk of Human Diseases Spread between Countries. Validation and Application to the 2014-2015 Ebola Virus Disease epidemic. Bulletin of Mathematical Biology, 77, 1668-1704. https://doi.org/10.1007/s11538-015-0100-x

[3] Ivorra, B., Ferrández, M.R., Vela-Pérez, M. and Ramos, A.M. (2020) Mathematical Modeling of the Spread of the Coronavirus Disease 2019 (COVID-19) Considering Its Particular Characteristics. The Case of China. Communications in Nonlinear Science and Numerical Simulation, 88, Article ID: 10503. 
https://doi.org/10.1016/j.cnsns.2020.105303

[4] Liu, Z., Magal, P., Seydi, O. and Webb, G. (2020) Predicting the Cumulative Number of Cases for the COVID-19 Epidemic in China from Early Data. Mathematical Biosciences and Engineering. https://doi.org/10.1101/2020.03.11.20034314

[5] Bacaër, N. (2020) Un modèle mathématique des débuts de l'épidémie de coronavirus en France. Mathematical Modelling of Natural Phenomena, 15, Article No. 29. https://doi.org/10.1051/mmnp/2020015

[6] European Centre for Disease Prevention and Control (2020) Discharge Criteria for Confirmed COVID-19 Cases-When Is It Safe to Discharge COVID-19 Cases from the Hospital or End Home Isolation?

https://www.ecdc.europa.eu/sites/default/files/documents/COVID-19-Discharge-cri teria.pdf

[7] Johns Hopkins University (JHU) (2020) Coronavirus COVID-19 Global Cases by the Center for Systems Science and Engineering (CSSE).

https://gisanddata.maps.arcgis.com/apps/opsdashboard/index.html/bda7594740fd4 $\underline{0299423467 \mathrm{~b} 48 \mathrm{e} 9 \mathrm{ecf6}}$

[8] Lin, Q.Y., Zhao, S., Gao, D.Z., et al. (2020) A Conceptual Model for the Coronavirus Disease 2019 (COVID-19) Outbreak in Wuhan, China with Individual Reaction and Governmental Action. International Journal of Infectious Diseases, 93, 211-216. https://doi.org/10.1016/j.ijid.2020.02.058

[9] Van den Driesche, P. and Watmough, J. (2002) Reproduction Numbers and Substhreshold Endemic Equilibria for the Compartmental Models of Disease Transmission. Mathematical Biosciences, 180, 29-48. https://doi.org/10.1016/S0025-5564(02)00108-6

[10] Liu, Z., Magal, P., Seydi, O. and Webb, G. (2020) Understanding Unreported Cases in the COVID-19 Epidemic Outbreak in Wuhan, China, and the Importance of Major Public Health Interventions. Biology, 9, 50. https://doi.org/10.3390/biology9030050

[11] Liu, T., Hu, J., Kang, M., Lin, L., Zhong, H., Xiao, J., et al. (2020) Transmission Dynamics of 2019 Novel Coronavirus (2019-nCoV). https://doi.org/10.2139/ssrn.3526307

[12] Li, R., Pei, S., Chen, B., Song, Y., Zhang, T., Yang, W. and Shaman, J. (2020) Substantial Undocumented Infection Facilitates the Rapid Dissemination of Novel Coronavirus (SARS-CoV2). Science, 368, 489-493.

https://doi.org/10.1126/science.abb3221 